\section{Papanicolaou na pós-menopausa: fatores associados a sua não realização}

\author{
Factors associated with lack of Pap smear \\ screening in a group of postmenopausal \\ Brazilian women
}

\author{
Sheila Cristina Rocha Brischiliari 1 \\ Cátia Millene Dell'Agnolo 1 \\ Laís Moraes Gil 1 \\ Tiara Cristina Romeiro 1 \\ Ângela Andréia França Gravena 1 \\ Maria Dalva de Barros Carvalho 1 \\ Sandra Marisa Pelloso ${ }^{1}$
}

\section{Introdução}

O câncer do colo do útero é um problema de saúde pública mundial 1 . Corresponde ao segundo câncer mais comum entre as mulheres no mundo, perdendo apenas para o câncer de mama 2,3. É considerado a principal causa de morte entre mulheres que vivem em países em desenvolvimento ${ }^{3}$.

Segundo dados da Agência Internacional de Pesquisas com Câncer, a incidência mundial do câncer do colo uterino no ano de 2008 foi de 530.232 novos casos, sendo que, desse total, 95.733 foram de mulheres com idade igual ou superior a 65 anos. A taxa de mortalidade para essa mesma faixa etária foi de 85.952 óbitos, de um total de 275.008 óbitos por câncer uterino ${ }^{4}$.

Apesar dos programas de rastreamento específicos para identificação e tratamento das lesões pré ou cancerígenas, a mortalidade por câncer do colo uterino continua alta em todo o mundo. Estimativas para o ano de 2020, segundo a Organização Mundial da Saúde (OMS), continuam a demonstrar dados alarmantes, com expectativa de 662.110 novos casos e 356.251 números de óbitos por essa causa. Desse total, a incidência para mulheres com idade igual ou superior a 65 anos será de 135.413, com uma mortalidade de 120.3915 .

No Brasil, segundo estimativas para 2012, o câncer do colo do útero terá 17.540 novos casos. Para a Região Sul, são esperados 2.000 novos ca- 
sos neste mesmo ano, e, no Estado do Paraná, 770 novos casos, com uma taxa bruta de 13,79 casos por 100 mil habitantes 6 . O número de óbitos no Brasil chegou a 5.063, em 20097.

A ocorrência do câncer de colo do útero é rara em mulheres de até 30 anos de idade, sendo que sua incidência aumenta gradativamente até alcançar seu pico nos 45-50 anos. Já a mortalidade aumenta consideravelmente a partir dos 40 anos de idade 6 .

Esse tipo de neoplasia apresenta um prognóstico bom, desde que seja diagnosticada e tratada precocemente ${ }^{8}$.

As estratégias utilizadas para a detecção precoce do câncer do colo do útero no Brasil compreendem o diagnóstico e o rastreamento precoce do câncer do colo do útero, seguindo a recomendação da OMS 9 . O método mais amplamente utilizado para esse rastreamento do câncer do colo do útero é o exame de Papanicolaou, realizado entre as mulheres com vida sexual ativa, com idade entre 25-59 anos. Com uma cobertura da população-alvo de $80 \%$, podese reduzir, em média, $60 \%-90 \%$ da incidência do câncer invasivo ${ }^{10}$. O Ministério da Saúde ainda preconiza que o exame seja realizado a cada três anos após dois resultados normais ou negativos para câncer, consecutivos, tendo intervalo de um ano 11.

Porém, merece atenção especial a repetição de exames por conveniência a cada ano, podendo ocasionar "confusão" nas taxas de cobertura divulgadas por órgão oficiais. Em um estudo realizado no Estado de São Paulo, mais de $50 \%$ dos exames foram realizados com um ano de intervalo, provavelmente de forma oportunística 12 . Assim, a cobertura trienal está muito prejudicada pelo excesso de exames em mulheres consideradas protegidas de acordo com as normas do Ministério da Saúde.

Houve uma redução de $80 \%$ da incidência do câncer do colo do útero, em países desenvolvidos, mediante um rastreamento citológico implantado com qualidade, cobertura e seguimento das mulheres 13 .

Considerando que o câncer de colo do útero está entre as prioridades do sistema público de saúde em nosso país, mantendo taxas inalteradas, a despeito de todo o empenho na melhora da prevenção e rastreamento dos últimos anos, acreditamos que a produção de dados e informações suplementares frente à cobertura e aos fatores determinantes para a sua ocorrência pode contribuir para a formulação de novas estratégias públicas de ação contra esse tipo de câncer, sendo realizada, portanto, a presente pesquisa com o objetivo de analisar os fatores de risco relacionados à não realização do exame de
Papanicolaou entre as mulheres que vivenciam a menopausa.

\section{Metodologia}

A presente pesquisa se caracteriza como um estudo de base populacional, composta de mulheres de 45-69 anos, residentes na área urbana de Maringá, Paraná, Brasil. Foram incluídas mulheres com cessação da menstruação há, pelo menos, 12 meses e com apresentação de menopausa natural. Esse último critério foi estabelecido a fim de garantir que as mulheres selecionadas encontrem-se, de fato, na fase da menopausa, uma vez que, com a retirada do útero, perde-se o parâmetro do cessar dos mênstruos. Essa opção também minimiza as diferenças entre os vários esquemas terapêuticos disponíveis. Foram excluídas mulheres diabéticas insulino-dependentes, hipertensas não controladas e com doenças da tireoide. Esse estudo faz parte de um inquérito populacional mais abrangente realizado com a finalidade de estudar fatores de risco relacionados ao câncer de mama e ao câncer de colo uterino, bem como fatores associados à não realização do rastreamento, em mulheres no período da pósmenopausa.

O tamanho da amostra foi estimado com o objetivo de assegurar representatividade para o evento. A amostra foi calculada utilizando as informações do Censo Demográfico referente à população feminina de idade entre 45-69 anos, relatada pelo Instituto Brasileiro de Geografia e Estatística (IBGE. http://www.ibge.gov.br/ho me/estatistica/populacao/censo2007, acessado 01/Abr/2009), no ano de 2000, para o Município de Maringá, e pela projeção da população estimada para o ano de 2009, perfazendo um total de 36.023 mulheres. Considerando um intervalo de 95\% de confiança (IC95\%) $(z=1,96)$, a estimativa da amostra deverá estar dentro de $\pm 5 \%$ (e) da real proporção da população de mulheres nessa faixa etária. Foi utilizado o modelo estatístico $\mathrm{n}=\mathrm{z}^{2}[\mathrm{p}(1-\mathrm{p})]: \mathrm{e}^{2}$ para populações infinitas $(\mathrm{N}>$ 10.000) que fornece o tamanho da amostra, calculado pelo programa estatístico Epi Info, versão 3.5.1 (Centers for Disease Control and Prevention, Atlanta, Estados Unidos), que, nesse caso, foi de 380 mulheres. Com um acréscimo de 20\%, para possíveis perdas e/ou recusas, o tamanho total da amostra foi de 456 mulheres.

Para o presente estudo, a seleção da amostra teve, como unidade de referência, os setores censitários do município, segundo o IBGE, tendo, como unidade amostral primária, os setores censitários, e os domicílios, como unidades amostrais secundárias. Utilizou-se a estratégia 
de amostragem sistemática com probabilidade proporcional ao tamanho do setor.

O Distrito de Maringá possui, segundo o IBGE (http://www.ibge.gov.br/home/estatistica/po pulacao/censo2007, acessado 01/Abr/2009), 407 setores censitários, sendo estudados 368 setores por serem caracterizados como áreas urbanizadas. Com a finalidade de obter uma representatividade de aleatoriedade, optou-se por adaptação da amostragem sistemática na seleção dos domicílios. A partir do croqui com os quarteirões numerados de cada setor censitário, o ponto de início do percurso e a esquina inicial de cada setor foram sorteados caminhando sempre em sentido horário. De cada setor, foi selecionada uma amostra aleatória simples, proporcional ao número de mulheres residentes em cada um desses setores, tendo em vista o tamanho da amostra (456).

Devido à quantidade de mulheres a ser pesquisada em cada setor ser proporcional ao tamanho da mesma e para proporcionar melhor distribuição para efeito de vizinhança, foi sorteado um domicílio e saltado três. Não coincidindo a casa escolhida com a presença da mulher, essa foi procurada no domicílio seguinte, reiniciandose o processo em cada entrevista. Na existência de mais de uma mulher no domicílio, foi realizado sorteio entre uma delas.

As visitas domiciliares incluíram a aplicação de um questionário face a face, após consentimento informado. Posteriormente à padronização, procedeu-se ao pré-teste do questionário, realizado em trinta mulheres da mesma faixa etária da pesquisa, em área de abrangência de uma Unidade Local de Saúde do Município. O estudo piloto foi realizado com trinta pessoas em um setor censitário sorteado dentre os não inclusos para o estudo propriamente dito.

A variável de desfecho primário foi a não realização autorreferida do exame de Papanicolaou nos últimos três anos para mulheres de 45-59 anos.

As medidas secundárias ou variáveis independentes avaliadas foram: idade (calculada em anos completos na data da entrevista); escolaridade (de acordo com a última série estudada, categorizada em até sete anos de estudo ou oito anos ou mais anos); estado civil (com ou sem companheiro); cor (dicotomizada em branca ou não branca); classe social (em classes econômicas A, B, C, D e de acordo com o critério de classificação econômica Brasil - Levantamento socioeconômico 2005. http:/ / ww.abep.org, acessado em 01/Abr/2009); ocupação (presença ou ausência de atividade remunerada); atividade física (avaliada de acordo com os critérios estabelecidos pela Sociedade Brasileira de Cardiolo- gia 14, sendo sedentária toda mulher sem atividade física regular, ou seja, com frequência mínima de três vezes por semana e duração não inferior a trinta minutos, independente da modalidade de exercício realizado); usuárias de Terapia de Reposição Hormonal - TRH (as mulheres foram questionadas quanto ao uso atual e ao tempo de uso de hormônios e classificadas em dois grupos: não usuárias de TRH, as que não fizeram uso de TRH nos últimos seis meses; e usuárias de TRH, as que fizeram uso contínuo por, pelo menos, seis meses; relato de consulta médica no último ano, consulta com o ginecologista, dificuldade de acesso ao sistema de saúde, realização de mamografia e exame clínico de mamas nos últimos dois anos.

Para análise estatística, foram utilizados o teste $\chi^{2}$ e o teste exato de Fisher, quando aplicável. As análises foram realizadas utilizando o software Epi Info 3.5.1, com nível de significância fixado em $\mathrm{p}<0,05$. Para a análise multivariada, realizada por meio da regressão logística, foram estudadas as variáveis independentes em relação ao uso de terapia de reposição hormonal, realizadas pelo programa Statistica 7.1 (Statsoft Inc.; http://www.statsoft.com), com nível de 5\% de significância.

O estudo foi aprovado pelo Comitê Permanente de Ética em Pesquisa com Seres Humanos da Universidade Estadual de Maringá (parecer $n^{\circ}$. 201/2010).

\section{Resultados}

Foram avaliadas 456 mulheres no período da menopausa, as quais apresentaram uma média de 58,7 $\pm 5,7$ anos, sendo 48,0 $\pm 5,0$ anos a idade média do início da menopausa. A prevalência de realização do exame de Papanicolaou foi verificada em $84,5 \%$ das pacientes estudadas. A maior parte $(56,6 \%)$ relatou ter até sete anos de estudo e apresentava companheiro (63,2\%); em relação à cor, 388 mulheres $(85,1 \%)$ eram brancas, sendo que $60,7 \%$ pertenciam à classe econômica $C$ e D, e 53,1\% das mulheres realizavam atividade remunerada. Diferenças foram apontadas em relação à escolaridade, havendo um aumento da não realização do Papanicolaou em mulheres com menor escolaridade, embora não significativo (Tabela 1).

Cerca de 327 (71,7\%) mulheres estudadas eram sedentárias; não usuárias de TRH 361 (79,2\%); $388(85,1 \%)$ realizaram consulta médica no último ano; e 432 (94,7\%) fizeram consulta com o ginecologista. A mamografia foi realizada pela maioria das mulheres (86\%). Quando questionadas sobre dificuldade de acesso ao sis- 
Características socioeconômicas e demográficas das mulheres menopausadas. Maringá, Paraná, Brasil, 2010.

\begin{tabular}{|c|c|c|c|c|}
\hline Variáveis & n (\%) & $\begin{array}{l}\text { Não realização de } \\
\text { Papanicolaou }\end{array}$ & $\begin{array}{c}\text { Razões de } \\
\text { prevalência (IC95\%) }\end{array}$ & Valor de $p$ \\
\hline \multicolumn{5}{|l|}{ Idade (anos) } \\
\hline $45-59$ & $245(53,7)$ & 15,5 & $1,31(0,74-2,31)$ & 0,32 \\
\hline $60 \mathrm{ou}+$ & $211(46,3)$ & 12,3 & 1,00 & \\
\hline \multicolumn{5}{|l|}{ Escolaridade (anos) } \\
\hline Até 7 & $258(56,6)$ & 16,7 & $1,69(0,93-3,06)$ & 0,06 \\
\hline $8 \mathrm{ou}+$ & $198(43,4)$ & 10,6 & 1,00 & \\
\hline \multicolumn{5}{|l|}{ Estado civil } \\
\hline Com companheiro & $288(63,2)$ & 11,8 & 1,00 & \\
\hline Sem companheiro & $168(36,8)$ & 17,9 & $1,62(0,92-2,86)$ & 0,07 \\
\hline \multicolumn{5}{|l|}{ Cor } \\
\hline Branca & $388(85,1)$ & 13,4 & 1,00 & \\
\hline Não branca & $68(14,9)$ & 17,6 & $1,38(0,65-2,88)$ & 0,35 \\
\hline \multicolumn{5}{|l|}{ Classe social } \\
\hline$A$ e $B$ & $179(39,3)$ & 9,5 & 1,00 & \\
\hline C e D & $277(60,7)$ & 17,0 & $2,19(11,17-4,11)$ & $<0,01 \star$ \\
\hline \multicolumn{5}{|l|}{ Ocupação } \\
\hline Remunerada & $242(53,1)$ & 9,5 & $0,44(0,25-0,79)$ & $<0,01 *$ \\
\hline Não remunerada & $214(46,9)$ & 19,2 & 1,00 & \\
\hline
\end{tabular}

IC95\%: intervalo de 95\% de confiança.

* Estatisticamente significativo ao nível de 5\%.

tema de saúde, $74,6 \%$ (340) delas responderam com negativa, assim como $86,4 \%$ (394) negaram história familiar de câncer de colo uterino (Tabela 2).

Após análise por regressão logística, foram associadas com a não realização do exame de Papanicolaou as variáveis idade, ocupação, consulta ao ginecologista e mamografia nos últimos dois anos (Tabela 3).

\section{Discussão}

Por ser parte integrante de uma pesquisa de inquérito populacional mais abrangente não realizada especificamente para coletar os dados em estudo, algumas questões importantes relacionadas aos fatores de risco para o câncer uterino, descritos na literatura, não foram estudadas, constituindo-se numa limitação deste estudo, embora não fosse o objetivo prioritário da pesquisa.

Cabe ressaltar que o objetivo deste estudo não foi verificar a ocorrência de fatores de risco para o câncer, mas, sim, para a não realização do seu rastreamento, o qual poderia proporcionar uma detecção precoce da doença.
O fato de o trabalho ser realizado somente na zona urbana acrescenta outra limitação, pois se acredita que essa população disponha de mais serviços que facilitam e incentivam a realização do exame de Papanicolaou. Por tratar-se de questionário com questões autorreferidas, esta pesquisa apresenta um viés de informação, já que o desconhecimento sobre o exame ou sua realização pode influenciar e gerar respostas inadequadas.

Em contrapartida, por ser um estudo de base populacional, permite identificar a real cobertura desse tipo de exame na população e não apenas o número de exames realizados pelo sistema público de saúde, além de buscar identificar fatores impedidores/redutores de sua realização.

O controle do câncer do colo do útero se destaca entre as políticas de saúde do Brasil. Em 1997, foi implantado, pelo Instituto Nacional de Câncer (INCA), o Programa de Prevenção do Câncer do Colo Uterino no Brasil, feito por meio de rastreamento pelo teste de Papanicolaou, e, em 2001, o seu controle foi incluído como uma das atividades inerentes à área de Saúde da Mulher 15.

A recomendação da OMS para cobertura de Papanicolaou é de rastreamento mínimo de 80\%- 
Tabela 2

Prevalência de não realização do exame Papanicolaou segundo comportamentos relacionados à saúde e morbidade. Maringá, Paraná, Brasil, 2010.

\begin{tabular}{|c|c|c|c|c|}
\hline Variáveis & $\mathrm{n}(\%)$ & $\begin{array}{c}\text { Não realização de } \\
\text { Papanicolaou }\end{array}$ & $\begin{array}{c}\text { Razões de } \\
\text { prevalência (IC95\%) }\end{array}$ & Valor de $p$ \\
\hline \multicolumn{5}{|c|}{ Atividade física } \\
\hline Sedentária & $327(71,7)$ & 16,2 & $2,07(1,01-4.37)$ & 0,03 * \\
\hline Ativa & $129(28,3)$ & 8,5 & 1,00 & \\
\hline \multicolumn{5}{|c|}{ Usuárias de TRH } \\
\hline Sim & $95(20,8)$ & 1,1 & 1,00 & \\
\hline Não & $361(79,2)$ & 17,5 & $19,87(2,92-390,96)$ & $<0,01$ * \\
\hline \multicolumn{5}{|c|}{ Relato de consulta médica } \\
\hline \multicolumn{5}{|l|}{ no último ano } \\
\hline Sim & $388(85,1)$ & 11,1 & 1,00 & \\
\hline Não & $68(14,9)$ & 30,9 & $3,58(1,87-6,83)$ & $<0,01$ * \\
\hline \multicolumn{5}{|c|}{ Relato de consulta com } \\
\hline \multicolumn{5}{|c|}{ ginecologista } \\
\hline Sim & $432(94,7)$ & 10,1 & 1,00 & \\
\hline Não & $24(5,3)$ & 16,7 & $44,09(13,4-160,3)$ & $<0,01$ * \\
\hline \multicolumn{5}{|c|}{ Realização de mamografia } \\
\hline Sim & $392(86,0)$ & 3,3 & 1,00 & \\
\hline Não & $64(14,0)$ & 42,7 & $21,70(10,38-46,7)$ & $<0,01$ * \\
\hline \multicolumn{5}{|c|}{$\begin{array}{l}\text { Dificuldade de acesso ao } \\
\text { sistema de saúde }\end{array}$} \\
\hline $\mathrm{Sim}$ & $116(25,4)$ & 16,4 & $1,28(0,68-2,38)$ & 0,40 \\
\hline Não & $340(74,6)$ & 13,3 & 1,00 & \\
\hline \multicolumn{5}{|c|}{ História familiar de câncer } \\
\hline \multicolumn{5}{|c|}{ de colo uterino } \\
\hline Sim & $62(13,6)$ & 8,1 & $0,52(0,12-1.83)$ & 0,27 \\
\hline Não & $394(86,4)$ & 14,6 & 1,00 & \\
\hline
\end{tabular}

IC95\%: intervalo de 95\% de confiança; OR: odds ratio; TRH: terapia de reposição hormonal.

* Estatisticamente significativo ao nível de $5 \%$.

Tabela 3

Análise multivariada para não realização de exame de Papanicolaou pelas mulheres na pós-menopausa de acordo com as variáveis independentes incluídas no modelo. Maringá, Paraná, Brasil, 2010.

\begin{tabular}{lccc}
\hline Variáveis & OR ajustada & IC95\% & Valor de p \\
\hline Idade (45-69 anos) & 3,11 & $1,35-7,15$ & $<0,01$ * \\
Escolaridade (até 7 anos) & 1,43 & $0,62-3,29$ & 0,38 \\
Classificação estado civil (sem companheiro) & 1,44 & $0,66-3,14$ & 0,35 \\
Classificação renda (classe C e D) & 1,63 & $0,70-3,79$ & 0,25 \\
Classificação cor (não branca) & 0,84 & $0,32-2,23$ & 0,74 \\
Ocupação (remunerada) & 0,33 & $0,14-0,75$ & $<0,01$ * \\
Classificação atividade física (sedentária) & 1,73 & $0,65-4,60$ & 0,27 \\
TRH (não usuárias) & 4,97 & $0,63-8,99$ & 0,12 \\
Sem consulta médica no último ano & 2,15 & $0,91-5,06$ & 0,07 \\
Sem consulta ao ginecologista & 38,54 & $8,83-68,13$ & $<0,01$ * \\
Sem realização de mamografia nos últimos 2 anos & 13,16 & $5,86-29,58$ & $<0,01$ * \\
Dificuldades de acesso & 1,09 & $0,49-2,45$ & 0,81 \\
História familiar de câncer de colo uterino & 0,84 & $0,17-4,12$ & 0,83 \\
\hline
\end{tabular}

IC95\%: intervalo de 95\% de confiança; OR: odds ratio; TRH: terapia de reposição hormonal.

* Significativo estatisticamente ao nível de $5 \%$. 
85\% da população feminina de 25-59 anos 13 . A cobertura entre as mulheres estudadas nesta pesquisa ficou próxima a esse valor, pois $84,5 \%$ delas realizaram o exame.

Embora não tenha sido encontrada significância estatística, as mulheres com menor escolaridade foram as que tiveram maior porcentagem de não realização do exame de Papanicolaou, mostrando similaridade com outros estudos $16,17,18,19,20,21$. Esse fato deve ser levado em consideração nas situações de rastreamento, pois a literatura descreve a baixa escolaridade como fator de risco para o desenvolvimento do câncer de colo uterino 20,22,23,24. Além disso, essa condição favorece o aparecimento de dificuldades sobre $o$ entendimento de medidas preventivas e fatores de risco 25 .

A ocorrência de maior número de parceiros sexuais é mais comum entre as mulheres solteiras, o que as expõe a carcinogênicos sexuais com maior frequência 26. Embora as mulheres sem companheiros fossem minoria em nosso estudo, a prevalência verificada de não realização de Papanicolaou foi maior entre elas.

As mulheres estudadas de cor branca foram a maioria deste estudo, porém as mulheres cuja cor referida foi não branca apresentaram maior prevalência de não realização do exame, embora não significativa, mesmo quando corrigida pelas outras variáveis. Isso denota a existência de desigualdade racial em relação ao acesso ao exame de Papanicolaou 18. Essa prevalência elevada também foi encontrada em outros estudos 18,19,21.

Outro fator que merece atenção especial foi a significância estatística na não realização do exame preventivo por mulheres pertencentes às classes sociais mais baixas (C e D). Menor renda familiar implica em maiores taxas de não realização do Papanicolaou 19,21. Em pesquisa realizada na zona urbana de São Leopoldo, as mulheres das classes C, D e E apresentaram maior prevalência de não realização do exame 27 . Dentre outras condições facilitadores para a ocorrência desse tipo de câncer, encontra-se o baixo nível socioeconômico, entre outras 22,28.

A não realização do exame de Papanicolaou foi maior entre as mulheres com ocupação não remunerada, todavia, as mulheres com atividade remunerada destacaram-se por terem apresentado esse fato como significante para a não realização do Papanicolaou ( $p=0,01)$. A mulher tem sido e deve ser vista cada vez mais como protagonista nas ações de prevenção do câncer do colo do útero, com agendas flexíveis que possibilitem a sua realização, de forma a diminuir desigualdades de acesso, trazendo mais participação, independente da sua condição trabalhista, ocupacional.
Em estudo realizado em Florianópolis, o número de mulheres com vida ativa $(45,6 \%)$ foi superior ao verificado em nosso estudo (28,3\%), e, em ambos, a prevalência de não realização do Papanicolaou foi maior entre as sedentárias 29.

A TRH não está associada ao câncer de colo uterino, porém, acredita-se que as mulheres menopausadas que fazem uso de TRH tenham acesso mais frequente e provável acompanhamento por profissional habilitado, constituindose em estímulo e oportunidade para a realização do Papanicolaou.

Em geral, a realização do Papanicolaou acompanha as atividades de rotina médica 30 , ida ao ginecologista, realização de outros exames nessa área, como a mamografia. Significância estatística foi verificada, com maiores chances de não realização do Papanicolaou, em mulheres que não realizaram consulta médica no último ano, corroborando com outros estudos 19,21,27. O fato de não procurar consulta médica de rotina eleva o risco de não procurar realizar o exame de Papanicolaou. Dessa forma, o médico clínico não tem oportunidade de induzir acompanhamentos específicos relativos à saúde da mulher, assim como não tem oportunidade de esclarecer a importância desse exame. Esse dado é demonstrado por outros autores $16 \mathrm{em}$ que os profissionais da saúde precisam aproveitar a oportunidade para coletar o exame citopatológico no momento da consulta, assim como estimular o retorno às consultas subsequentes.

A presença de queixas ginecológicas foi o motivador para a realização do último exame de Papanicolaou em outra pesquisa $(18,2 \%)$. Isso reporta a outro questionamento de que as pessoas somente procuram atendimento médico na presença de sintomas clínicos evidentes e não como prevenção 21,31. Há um desconhecimento da população sobre a importância do screening como método preventivo.

Em pesquisa realizada no Município de São Paulo, foram encontrados, como fatores associados à não realização do exame citológico, o relato de boa saúde ginecológica pelas mulheres; vergonha e desconforto; atendimento insuficiente com dificuldades para marcar vagas, respectivamente, relatados por $45 \%, 32 \%$ e $11 \%$ das mulheres, e, em menor porcentagem, a não realização do exame foi atribuída a dificuldades financeiras, transporte e falta de tempo, falta de interesse, ausência de recomendação médica, desconhecimento sobre o exame e outros 31 .

Não ter ido à consulta com ginecologista e não ter realizado mamografia também mostraram associação estatística significativa em nosso estudo para a não realização preventiva do Papanicolaou. Isso demonstra a necessidade de 
expandir o rastreamento desse exame visando melhorar a assistência à mulher nas unidades de saúde, por meio da melhoria dos serviços de busca ativa e acompanhamento pelo programa saúde da família.

Atenção especial tem que ser dada ao fato de que mesmo algumas mulheres que tiveram contato com profissionais médicos (consulta médica ou ginecologista) não realizaram o exame, demonstrando haver uma provável falha no programa de rastreamento, pois esse contato poderia e deveria ser utilizado também para aumentar a adesão ao programa, visando à melhor assistência à saúde da mulher.

Em estudo realizado em duas cidades brasileiras (Fortaleza e Rio de Janeiro), entre as maiores razões de prevalência para a não realização do exame de Papanicolaou, estavam as mulheres que nunca realizaram mamografia anteriormente 32 . Embora, em nossa pesquisa, a porcentagem de mulheres que não realizaram mamografia nos últimos dois anos tenha sido baixa (14\%), a razão de prevalência de não realização do Papanicolaou foi elevada.

Em outra pesquisa nacional, realizada com mulheres de faixa etária próxima deste estudo (35-49 anos), mais da metade delas relataram a procura espontânea como motivo para a realização do último exame de Papanicolaou 31. Chama a atenção o fato de essa faixa etária ser priorizada pelos programas de prevenção ao câncer cérvico-uterino.

Em detrimento dos fatores de risco verificados neste estudo, colocados como "responsáveis” pela não realização do exame de prevenção ao câncer de colo de útero, a maioria (57\%) das 264 mulheres pesquisadas, residentes num município de Santa Catarina, relatou não ter dificuldades na realização do Papanicolaou, seguida por $23 \%$ delas que relatavam a presença de nervosismo/vergonha, e $9 \%$ associavam a coleta à dor e ao desconforto na coleta 33. Isso denota a necessidade de uma assistência voltada à educação dessas mulheres, com melhora na adesão de medidas de prevenção e promoção da saúde.

\section{Conclusão}

As mulheres desta pesquisa apresentavam escolaridade e classe social baixas, fatores de risco conhecidos para o câncer de colo de útero.

As participantes que possuíam ocupação remunerada, não eram usuárias de TRH, não passaram por consulta médica no último ano, não consultaram ginecologista e que não realizaram mamografia foram as que menos procuraram realizar o exame preventivo de colo do útero (significativo estatisticamente).

Os achados evidenciaram a necessidade de intervenção em forma de educação em saúde para conscientização das mulheres que não realizam o exame de Papanicolaou, pois, independentemente da existência de fatores de risco, deve ser estimulada a prevenção. Para isso, as informações obtidas neste e em outros estudos sobre os fatores associados à não realização desse exame podem ser utilizadas para se programar estratégias direcionadas a atingir as mulheres que não procuram assistência médica preventiva, promovendo o acesso de todos os grupos, alcançando, assim, as mais vulneráveis.

Os esforços para melhorar o rastreamento do câncer de colo do útero devem estar focados no conhecimento das mulheres e na diminuição dos fatores contribuintes para a não realização do exame de Papanicolaou. 


\section{Resumo}

O objetivo deste estudo foi analisar os fatores de risco relacionados à não realização do exame de Papanicolaou entre as mulheres que vivenciam a menopausa. Neste estudo de base populacional, composto de mulheres de 45-69 anos, residentes na área urbana de Maringá, Paraná, Brasil, foram avaliadas $456 \mathrm{mu}$ lheres no período da menopausa, as quais apresentaram uma média de 58,7 \pm 5, 7 anos, sendo 48,0 \pm 5,0 anos a idade média do início da menopausa. A maior parte relatou ter até sete anos de estudo, apresentava companheiro, realizava atividade remunerada, era sedentária e não usuária de terapia de reposição hormonal. A cobertura de rastreamento de Papanicolaou foi de 84,5\%. Após ajuste por análise multivariada, a faixa etária de 45-69 anos, ocupação remunerada, sem consulta ao ginecologista no último ano e sem realização de mamografia nos últimos dois anos foram associadas à não realização do exame. Os esforços para melhorar o rastreamento do câncer de colo do útero devem estar focados no conhecimento das mulheres e na diminuição dos fatores contribuintes para a não realização do exame de Papanicolaou.

Esfregaço Vaginal; Neoplasias do Colo do Útero; Saúde da Mulher; Programas de Rastreamento

\section{Colaboradores}

S. C. R. Brischiliari colaborou na redação, coleta, estatística, análise e discussão. C. M. Dell'Agnolo contribuiu na redação, estatística, análise de dados e discussão. L. M. Gil participou na coleta de dados e redação do artigo. T. C. Romeiro contribuiu na redação, coleta de dados. A. A. F. Gravena participou na coleta de dados, estatística e redação. M. D. B. Carvalho e S. M. Pelloso colaboraram na redação e revisão do artigo.

\section{Referências}

1. Ribeiro LP, Maradei CM, Silva CL, Tombolato RM, Vieira EM. Prevenção de câncer de colo uterino em uma área do programa saúde da família em Ribeirão Preto. Revista APS 2004; 7:91-5.

2. Bueno KS. Atipias escamosas de significado indeterminado: novas qualificações e importância na conduta clínica. Rev Bras Anal Clín 2008; 40:121-8.

3. Instituto Nacional de Câncer. Estimativa 2008 - incidência de câncer no Brasil. http://www.inca.gov. br/estimativa/2008versaofinal.pdf (acessado em 13/Mai/2011).

4. International Agency for Research on Cancer. Cancer incidence in five continents. v. I to IX. http: http://globocan.iarc.fr/factsheet.asp\#WOMEN (acessado em 04/Mar/2012).
5. World Health Organization/International Agency for on Cancer. Globocan 2008. Cancer incidence, mortality and prevalence worldwide in 2008. Online Analysis. Prediction, 2010. http://www.cancer. eaea.org/ (acessado em 13/Mar/2012).

6. Instituto Nacional de Câncer. Estimativa 2012: incidência de câncer no Brasil. http://wwwwlinca. gov.br/estimativa/2012/tabelaestados.asp?UF=BR (acessado em 04/Mar/2012).

7. Instituto Nacional de Câncer. Tipos de câncer: colo do útero. http://www2.inca.gov.br/wps/wcm/ connect/tiposdecancer/site/home/colo_utero/ definicao (acessado em 04/Mar/2012). 
8. Instituto Nacional de Câncer. Inquérito domiciliar sobre comportamentos de risco e morbidade referida de doenças e agravos não transmissíveis: Brasil, 15 capitais e Distrito Federal, 2002-2003. Rio de Janeiro: Instituto Nacional de Câncer; 2004.

9. World Health Organization. Cancer control. Knowledge into action. WHO guide for efective programmes. http://www.who.int/cancer/modules/ Prevention\%20Module.pdf (acessado em 02/Abr/ 2009).

10. World Health Organization. National cancer control programmes: policies and managerial guidelines. 2nd Ed. Geneva: World Health Organization; 2002.

11. Coordenação de Prevenção e Vigilância, Instituto Nacional de Câncer. Nomenclatura brasileira para laudos cervicais e condutas preconizadas - recomendações para profissionais de saúde. Rio de Janeiro: Instituto Nacional de Câncer; 2006.

12. Vale DBAP, Morais SS, Pimenta AL, Zeferino LC. Avaliação do rastreamento do câncer do colo do útero na Estratégia Saúde da Família no Município de Amparo, São Paulo, Brasil. Cad Saúde Pública 2010; 26:383-90.

13. World Health Organization. Manual on the prevention and control of common cancers. Geneva: World Health Organization; 1998. (Westerns Pacific Series, 20).

14. Sposito AC, Caramelli B, Fonseca FA, Bertolami MC, Afiune Neto A, Souza AD, et al. IV Diretriz Brasileira Sobre Dislipidemias e Prevenção da Aterosclerose. Departamento de Aterosclerose da Sociedade Brasileira de Cardiologia. Arq Bras Cardiol 2007; 88 Suppl 1:1-19.

15. Ministério da Saúde. Portaria no ${ }^{\circ}$ 399/GM de 22 de fevereiro de 2006. Divulga o Pacto pela Saúde - Consolidação do SUS e aprova as Diretrizes Operacionais do Referido Pacto. Diário Oficial da União 2006; 23 fev.

16. Hackenhaar AA, Cesar JA, Domingues MR. Exame citopatológico de colo uterino em mulheres com idade entre 20 e 59 anos em Pelotas, RS: prevalência, foco e fatores associados à sua não realização. Rev Bras Epidemiol 2006; 9:103-11.

17. Oliveira MMHN, Silva AAM, Brito LMO, Coimbra LC. Cobertura e fatores associados à não realização do exame preventivo de Papanicolaou em São Luís, Maranhão. Rev Bras Epidemiol 2006; 9: 325-34.

18. Amorim VMSL, Barros MBA, Cesar CLG, Carandina $\mathrm{L}$, Goldbaum M. Fatores associados à não realização do exame de Papanicolaou: um estudo de base populacional no Município de Campinas, São Paulo, Brasil. Cad Saúde Pública 2006; 22:2329-38.

19. Quadros CAT, Victora CG, Dias-da-Costa JS. Coverage and focus of a cervical cancer prevention program in Southern Brazil. Rev Panam Salud Pública 2004;16:223-38

20. Cesar JA, Horta BL, Gomes G, Houlthusen RS, Willrich RM, Kaercher A, et al. Fatores associados à não realização de exame citopatológico de colo uterino no extremo Sul do Brasil. Cad Saúde Pública 2003;19:1365-72.
21. Dias-da-Costa JS, Olinto MTA, Gigante DP, Menezes AMB, Macedo S, Borba AT, et al. Cobertura do exame citopatológico na cidade de Pelotas, Rio Grande do Sul, Brasil. Cad Saúde Pública 2003; 19:191-7.

22. Rodrigues Neto JF, Figueiredo MFS, Siqueira LG. Exame citopatológico do colo do útero: fatores associados à não realização em ESF. Rev Eletrônica Enferm 2008; 10:610-21.

23. Castro-Jiménez MA, Vera-Cala LM, Posso-Valencia HJ. Epidemiología del cáncer de cuello uterino: estado del arte. Rev Colomb Obstet Ginecol 2006; 57:182-9.

24. Departamento de Atenção Básica, Secretaria de Atenção à Saúde, Ministério da Saúde. Controle dos cânceres do colo do útero e de mama. Brasília: Ministério da Saúde; 2006.

25. Santos MS, Macedo APN, Leite MAG. Percepção de usuárias de uma unidade de saúde da família acerca da prevenção do câncer do colo do útero. Revista APS 2010; 13:310-9.

26. Pinto IC, Oliveira MM. Percepção das usuárias sobre as ações de prevenção do câncer do colo do útero na estratégia saúde da família em uma distrital de saúde do Município de Ribeirão Preto, São Paulo, Brasil. Rev Bras Saúde Matern Infant 2007; 7:31-8.

27. Muller DK, Dias-da-Costa JS, Luz AMH, Olinto MTA. Cobertura do exame citopatológico do colo do útero na cidade de São Leopoldo, Rio Grande do Sul, Brasil. Cad Saúde Pública 2008; 24:2511-20.

28. Drain PK, Holmes KK, Hughes JP, Koutsky LA. Determinants of cervical cancer rates in developing countries. Int J Cancer 2002; 100:199-205.

29. Gasperin SI, Boing AF, Kupek E. Cobertura e fatores associados à realização do exame de detecção do câncer de colo do útero em área urbana no Sul do Brasil: estudo de base populacional. Cad Saúde Pública 2011; 27:1312-22.

30. Albuquerque KM, Frias PG, Andrade CLT, Aquino EML, Menezes G, Szwarcwald CL. Cobertura do teste de Papanicolaou e fatores associados à não realização: um olhar sobre o Programa de Prevenção do Câncer do Colo do Útero em Pernambuco, Brasil. Cad Saúde Pública 2009; 25 Suppl 2:S301-9.

31. Pinho AA, França Junior I, Schraiber LB, D'Oliveira AFPL. Cobertura e motivos para a realização ou não do teste de Papanicolaou no Município de São Paulo, Brasil. Cad Saúde Pública 2003; 29 Suppl 2:S303-13.

32. Martins LFL, Valente JG, Thuler LCS. Fatores associados ao rastreamento inadequado do câncer cervical em duas capitais brasileiras. Rev Saúde Pública 2009; 43:318-25.

33. Feliciano C, Christen K, Velho MB. Câncer de colo uterino: realização do exame colpocitológico e mecanismos que ampliam sua adesão. Rev Enferm UERJ 2010; 18:75-9.

Recebido em 26/Mar/2012

Versão final reapresentada em 09/Jul/2012 Aprovado em 13/Jul/2012 